\title{
Unusually stable helical coil allotrope of phosphorus
}

\author{
Dan Liu ${ }^{\dagger}$ Jie Guan, ${ }^{\dagger}$ Jingwei Jiang, ${ }^{\ddagger}$ and David Tománek $^{*, \dagger}$ \\ Physics and Astronomy Department, Michigan State University, East Lansing, Michigan 48824, \\ USA, and Department of Physics, Peking University, Beijing, 100871, China \\ E-mail: tomanek@pa.msu.edu
}

KEYWORDS: phosphorus, stability, helical coil, ab initio calculations, electronic structure

\section{Abstract}

We have identified an unusually stable helical coil allotrope of phosphorus. Our ab initio Density Functional Theory calculations indicate that the uncoiled, isolated straight $1 \mathrm{D}$ chain is equally stable as a monolayer of black phosphorus dubbed phosphorene. The coiling tendency and the attraction between adjacent coil segments add an extra stabilization energy of $\approx 12 \mathrm{meV} /$ atom to the coil allotrope, similar in value to the $\approx 16 \mathrm{meV} /$ atom inter-layer attraction in bulk black phosphorus. Thus, the helical coil structure is essentially as stable as black phosphorus, the most stable phosphorus allotrope known to date. With an optimum radius of $2.4 \mathrm{~nm}$, the helical coil of phosphorus may fit well and even form inside wide carbon nanotubes.

Elemental phosphorus has been known for its unusual properties since its isolation as the white phosphorus allotrope, 1 a $\mathrm{P}_{4}$-based molecular solid, in 1669. Other observed allotropes include violet phosphorus,, 233 also known as Hittorf's metallic phosphorus, ${ }^{45}$ rather common fibrous red phosphorus ${ }^{516}$ with an amorphous structure, and layered black phosphorus, ${ }^{77}$ known as the most stable crystalline allotrope. Other bulk

\footnotetext{
*To whom correspondence should be addressed

†Physics and Astronomy Department, Michigan State University, East Lansing, Michigan 48824, USA

†Permanent address:
}

allotropes, including blue phosphorene, have been predicted $^{8}$ and subsequently synthesized. ${ }^{9}$ Other structures of elemental phosphorus, which have been studied, include $\mathrm{P}_{n}$ clusters ${ }^{10-12]}$ and atomically thin $\mathrm{P}$ helices, which have been identified as constituents ${ }^{13}$ in the complex structure of SnIP. It thus appears quite possible that still more allotropes may be synthesized in the future.

We report here theoretical results that identify an unusually stable helical coil allotrope of phosphorus. Our ab initio Density Functional Theory calculations indicate that the uncoiled, isolated straight $1 \mathrm{D}$ chain is equally stable as a monolayer of black phosphorus dubbed phosphorene. The coiling tendency and the attraction between adjacent coil segments add an extra stabilization energy of $\approx 12 \mathrm{meV} /$ atom to the coil allotrope, similar in value to the $\approx 16 \mathrm{meV} /$ atom inter-layer attraction in bulk black phosphorus. Thus, the helical coil structure is essentially as stable as black phosphorus, the most stable phosphorus allotrope known to date. With an optimum radius of $2.4 \mathrm{~nm}$, the helical coil of phosphorus may fit well and even form inside wide carbon nanotubes.

\section{Equilibrium structure and sta- bility results}

The unusually stable structure of a $\mathrm{P}_{10}$ cluster and its suitability to link up to an infinite 1D chain was discovered while developing and testing a $\mathrm{Ge}$ netic Algorithm optimization technique for phosphorus clusters based on a tight-binding formalism. ${ }^{14}$ The optimum structure of a $\mathrm{P}_{10}$ unit cell in a straight $1 \mathrm{D}$ chain, which resembles a narrow 
(a)

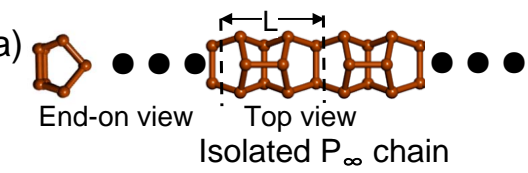

(c)

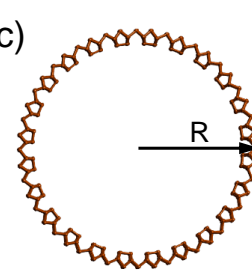

End-on view $P_{\infty}$ helical coil

(d)

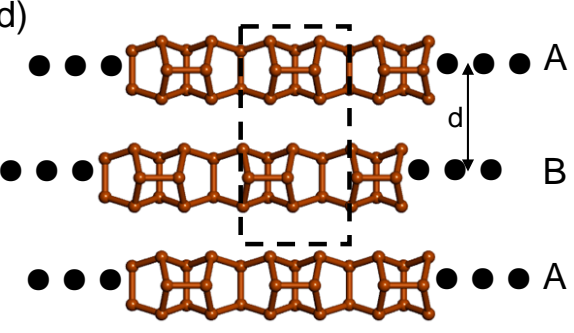

Layer of $\mathrm{P}_{\infty}$ chains (b)

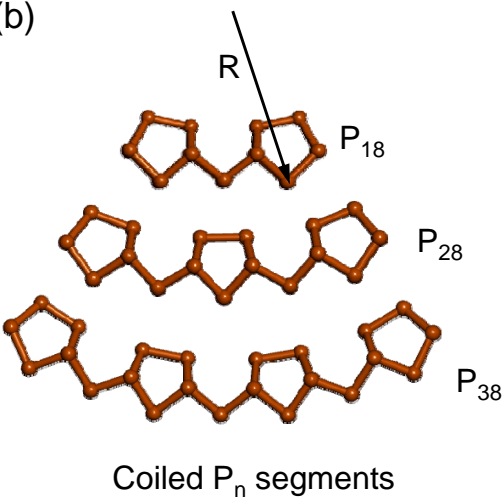

(e)

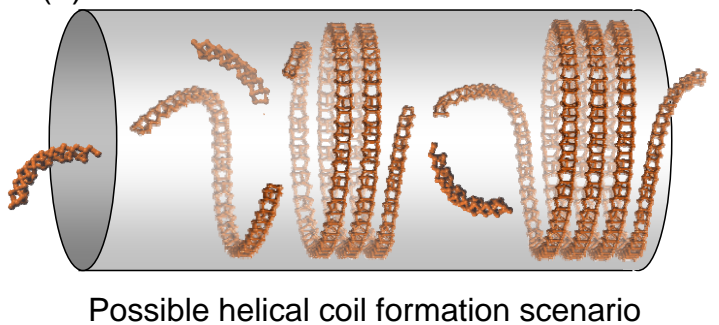

Figure 1: (Color online) (a) Optimum structure of an isolated, straight $1 \mathrm{D} \mathrm{P}_{\infty}$ chain with the $\mathrm{P}_{10}$ unit cell of length $L$. (b) Optimized $\mathrm{P}_{18}, \mathrm{P}_{28}$ and $\mathrm{P}_{38}$ segments of the isolated chain, indicating the tendency to form rings with a radius $R \approx 2.4 \mathrm{~nm}$. (c) The optimum structure of a single coil. (d) Optimum structure of a 2D assembly of $\mathrm{P}_{\infty}$ chains separated by the distance $d$. (e) Possible scenario for the formation of helical coils by connecting finite-length chain segments inside a cylindrical cavity. The unit cells are indicated by dashed lines in (a) and (d).

tube with a pentagonal cross-section, is shown in Fig. 1(a). We notice a structural similarity with fibrous red ${ }^{5 / 6}$ and violet phosphorus ${ }^{2-5}$ structures, which also contain $\mathrm{P}_{10}$ subunits in the interlinked chains. The postulated chain structure is also similar to $\mathrm{P}$ nanorods $\frac{15 \mid 16}{16}$ and $\mathrm{P}$ tubes ${ }^{17}$ observed in the $\mathrm{AgP}_{15}$ compound. Our DFT-PBE calculations indicate a binding energy $E_{c o h}=3.274 \mathrm{eV} /$ atom for the postulated $\mathrm{P}_{10}$ structure with respect to spin-polarized $\mathrm{P}$ atoms. This value is only negligibly larger than that of a monolayer of black phosphorus, known as the most stable phosphorus allotrope, with $E_{c o h}=3.273 \mathrm{eV} /$ atom.

Finite chain segments, shown in Fig. 11(b), display a tendency to form coils with an average radius of $2.4 \mathrm{~nm}$. We find this coiling, which had been identified earlier, $10[18$ to be associated with an energy gain of $6 \mathrm{meV} /$ atom (PBE) and 9 meV/atom (LDA). Assuming that an ordered $\mathrm{P}_{\infty}$ system may form by connecting finite $\mathrm{P}_{n}$ segments end-to-end, the resulting equilibrium structure will be a helical coil, depicted in Fig. 1(c). On a per-atom basis, the elastic strain $\Delta E$ in the coil is shown in Fig. 2(a) as a function of radius $R$. The data points are well represented by

$$
\Delta E=\frac{1}{2} k\left(\frac{1}{R}-\frac{1}{R_{e q}}\right)^{2},
$$

where $R_{e q}$ is the equilibrium radius. This expression describes the local strain energy in a finitelength 1D beam thought to be initially aligned with the $x$-direction and deformed to a circular arc of radius $R$ in the $x z$-plane. The local strain is $\sigma=d^{2} u_{z} / d x^{2}=1 / R$. Should not a straight, but rather a bent beam of radius $R_{e q}$ represent the equilibrium structure, then the local strain would be $\sigma=d^{2} u_{z} / d x^{2}-1 / R_{e q}=1 / R-1 / R_{e q}$. Equation (??) describes the corresponding local strain energy. ${ }^{19}$ We find $k=7.2 \mathrm{eV} \AA^{2}$ for the rigidity of the elastic beam and $R_{e q}=24 \AA$ for the optimum radius of curvature based on PBE. The LDA values of $k=7.5 \mathrm{eV}^{2}$ and $R_{e q}=21 \AA$ are in fair agreement with the PBE values. 
(a)

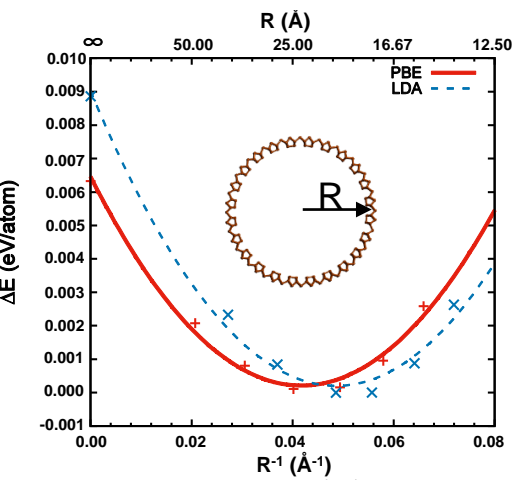

(b)

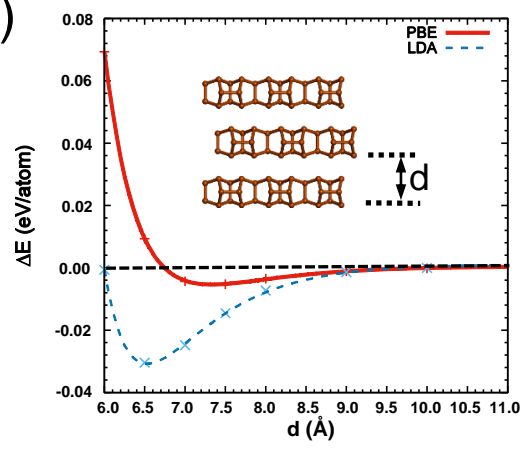

(c)

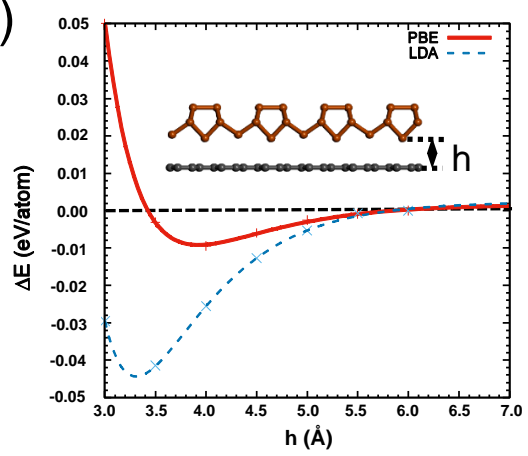

Figure 2: (Color online) (a) Strain energy $\Delta E$ per atom as a function of the radius $R$ of an isolated $\mathrm{P}$ coil. (b) Inter-chain interaction energy $\Delta E$ per atom in a 2D assembly of phosphorus chains, depicted in Fig. 11(d), as a function of the inter-chain distance $d$. (c) Interaction energy $\Delta E$ per phosphorus atom between a $\mathrm{P}$ chain and a graphene monolayer as a function of the adsorption height $h$. PBE results are shown by the solid red lines, LDA results by the dashed blue lines.

Same as in the infinite chain, the stability of the helical coil is dominated by the covalent interatomic bonds, which are described well by DFT calculations. The coil is further stabilized by the weak attraction between neighboring strands that is similar in nature to the inter-layer attraction in bulk black phosphorus. As shown in superior Quantum Monte Carlo (QMC) calculations of the latter system, 20 the fundamental nature of the inter-layer interaction is rather non-trivial, different from a van der Waals interaction, and not reproduced well by DFT functionals with or without van der Waals corrections. When compared to the more accurate QMC value of $81 \mathrm{meV} /$ atom, the LDA value of $94 \mathrm{meV} /$ atom overestimates and the PBE value of $16 \mathrm{meV} /$ atom underestimates the inter-layer interaction in bulk black phosphorus. We also notice the large ratio of $5-6$ between PBE and LDA values for the weak inter-layer interaction. Extrapolating what is known about the interlayer interaction in black phosphorus to the inter-chain interaction in a 2D assembly of chains of Fig. 11(d) or the related wall of the helical coil in Fig. 1(c), we expect that PBE will also underestimate and LDA overestimate the value of this weak interaction.

In view of the fact that the optimum coil radius is much larger than the chain thickness, the wall of the infinite helical coil in Fig. 11(c) is well represented by a 2D assembly of chains of Fig. 11(d). We found that the most stable 2D arrangement is one with $A B$ stacking of chains. The inter-chain interaction energy $\Delta E$ is displayed as a function of the inter-chain distance $d$ in Fig. 2(b). As anticipated above, we expect a large difference between PBE and LDA interaction energies. We obtain the optimum distance $d_{e q}=7.3 \AA$ and the interaction energy $\Delta E=5.3 \mathrm{meV} /$ atom based on PBE. LDA suggests a smaller separation $d_{e q}=$ $6.5 \AA$ and a much larger interaction energy $\Delta E=$ $30.8 \mathrm{meV} /$ atom. While still small, the LDA interaction energy is roughly five times higher than the PBE value.

\section{Likely synthesis scenario}

Postulating a new allotrope is of limited use without a plausible formation scenario. We note that previously, the void inside carbon nanotubes has been successfully filled by sublimed $\mathrm{C}_{60}$ fullerenes that eventually fused to an inner nanotube. 21 Similarly, functionalized diamondoid molecules were observed to enter the nanotube void, where they converted to carbon chains ${ }^{22}$ or diamond nanowires. ${ }^{23}$ Inspired by these results, we feel that the most suitable scenario to form a helical coil phosphorus allotrope involves a cylindrical cavity, 
(a)
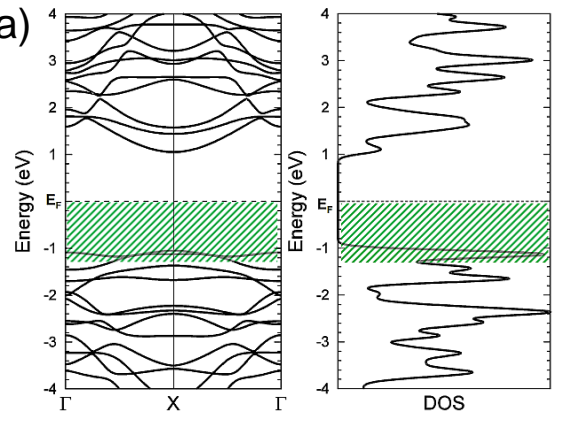

(b)
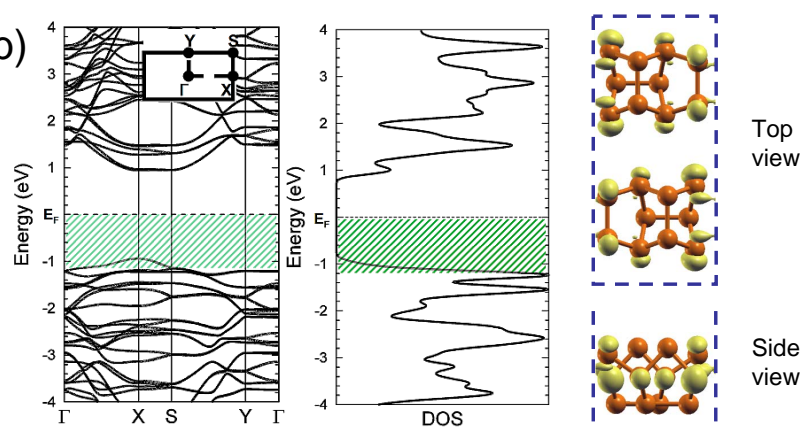

Figure 3: (Color online) Electronic structure of (a) an isolated $\mathrm{P}$ chain and (b) a 2D layer of $\mathrm{P}$ chains, shown in Figs. 1(a) and 1(d). Left panels depict the electronic band structure based on PBE and the middle panels the corresponding density of states. The Brillouin zone is shown as inset of the left panel in (b). The right panels depict the charge distribution associated with frontier states in the valence band region, indicated by the green hashed region in (a) and (b), which extends from $E_{F}$ to $0.2 \mathrm{eV}$ below the top of the valence band. Charge density contours are superposed to structural models, with the unit cells indicated by the dashed lines. Due to differences in the density of states between these systems, the contours are presented at the electron density $0.008 \mathrm{e} / \AA^{3}$ in (a) and $0.002 \mathrm{e} / \AA^{3}$ in (b).

shown in Fig. 11(e).

Suitable cavities with an optimum inner diameter of few nanometers may be found in zeolites or in nanotubes of carbon, $\mathrm{BN}$ and other materials. The phosphorus feedstock could be white, red or violet phosphorus that had been sublimed in vacuum, under exclusion of air. The sublimed species would likely be finite-chain segments, shown in Fig. 1(b), which may enter at the open end and benefit energetically from the interaction with the inner wall of the cavity. As seen in Fig. 2(c), where we consider the related system of an isolated chain on graphene, this interaction is weak and similar in nature to the inter-chain interaction in Fig. 2(b). The optimum arrangement is found by inspecting the adsorption energy $\Delta E$ as a function of height $h$ in Fig. 2(c). For the optimum geometry, we find $\Delta E_{e q}=9 \mathrm{meV}$ per $\mathrm{P}$ atom at $h_{e q}=3.9 \AA$ based on $\mathrm{PBE}$ and $\Delta E_{e q}=44 \mathrm{meV}$ per $\mathrm{P}$ atom at $h_{e q}=3.3 \AA$ based on LDA. We notice here again the adsorption energy ratio of $\approx 5$ between LDA and PBE results, consistent with our other results.

Once inside and near the wall of the cylindrical cavity, finite $\mathrm{P}_{n}$ chain fragments will benefit energetically from an end-to-end connection that eliminates open ends. The number of atoms in the finite circular arc, preferentially oriented along the perimeter of the inner cavity, will grow. At the elevated temperatures of subliming phosphorus, the growing $\mathrm{P}_{n}$ ring fragments are very unlikely to interconnect with corresponding segments that contain exactly the right number of atoms, which would complete a ring at the optimum distance to the wall. Much more likely, the last segment to join before possible ring closure will be too long and start the formation of a helical coil. Since transformation of the coil to one or more adjacent rings would require bond breakage within the coil, it is unlikely to happen.

\section{Electronic structure results}

The electronic structure of the new allotrope, similar to that of phosphorene, is of utmost interest. 24 Our PBE results for the related phosphorus chains and their 2D assemblies are shown in Fig. 3. As seen in Fig. 3(a), the $\mathrm{P}_{\infty}$ chain has a direct fundamental band gap of $2.03 \mathrm{eV}$ at $X$. Similarly, also the $2 \mathrm{D}$ chain assembly has a direct band gap of $1.82 \mathrm{eV}$ at $X$ as seen in Fig. 3(b). Based on what is known theoretically and experimentally about few-layer phosphorene, 24 the PBE band gap values are strongly underestimated in comparison to the experiment.

In view of the fact that phosphorus is typically $p$-doped, we are also interested in the nature of the frontier states in the valence band region. We plotted the charge distribution of these states, covering the energy range between $E_{F}$ and $0.2 \mathrm{eV}$ below the top of the valence band, in the right panels of Fig. 3. Similar to what is known about black 
phosphorus, we observe lone-pair electron states in the isolated chain in Fig. 3 (a) that contributes to the electronic inter-chain coupling modifying the band structure, as seen in Fig. 3(b). This demonstrated influence of the inter-chain coupling on the electronic structure is a clear evidence that the interaction differs from a purely van der Waals interaction, similar to black phosphorus. 20

\section{Discussion}

In view of the relatively low beam rigidity of the coiling chain, we expect the coils to adjust their radius freely for the optimum fit inside cylindrical cavities. The ability of the helical coil strands to slide past each other allows the helix to adjust to a changing cavity diameter. In view of the favorable inter-chain interaction of $16 \mathrm{meV} / \mathrm{atom}$ for an $\mathrm{AB}$ stacking in the radial direction, with an optimum inter-chain distance of $0.6 \mathrm{~nm}$ based on PBE, we consider it quite possible for a second helix forming inside the outer helix. Considering an outer helical coil at its equilibrium radius $R_{\text {out }}=2.4 \mathrm{~nm}$, the inner coil should have a radius of $R_{i n} \approx 1.8 \mathrm{~nm}$. In PBE, the strain energy in the inner structure of $<1 \mathrm{meV} /$ atom according to Fig. 2(a) is negligibly small when compared to the additional interchain interaction energy of $16 \mathrm{meV} / \mathrm{atom}$. We may even imagine additional helices forming inside the double-helix structure. In view of the low beam rigidity of the chain and the large inter-chain interaction, a structure consisting of nested coaxial coils should be even more stable than bulk black phosphorus, the most stable phosphorus allotrope known to date.

As suggested by the end-on view of a chain in Fig. 1 (a), the cross-section of the helical coil should appear as lines of pentagons near the walls and along the axis of the cylindrical cavity in Transmission Electron Microscopy (TEM) images. This is very similar to recently observed TEM data. ${ }^{25}$ We can imagine left- and righthanded helical coils forming simultaneously and coexisting inside a suitable cylindrical cavity.

As a structural alternative, a black phosphorus monolayer may also roll up to a tube inside a carbon nanotube with a $2.4 \mathrm{~nm}$ radius. Assuming an inter-wall distance of $0.5 \mathrm{~nm}$, the radius of the phosphorene nanotube should be $R \approx 1.9 \mathrm{~nm}$, and its strain energy should be $\approx 8.6 \mathrm{meV} / \mathrm{atom}$ if bent along the soft direction to become an armchair $\mathrm{P}$ nanotube, or $42.1 \mathrm{meV} / \mathrm{atom}$ if bent along the normal, harder direction. ${ }^{26}$ Thus, energetically, a black phosphorus nanotube is not favorable. Also a straight $1 \mathrm{D}$ phosphorus chain inside a nanotube should be less stable by $>6 \mathrm{meV} / \mathrm{atom}$ than the coiled structure. Thus, we find the coil to be more stable than competing phosphorene nanotube and straight chain structures. Also, assuming that the new phase forms by sublimation of red phosphorus, it will more likely resemble structural elements of red $\mathrm{P}$ than the completely different black $\mathrm{P}$.

In layered black phosphorus, the observed bulk band gap value of $0.35 \mathrm{eV}$ is known to increase to $2 \mathrm{eV}$ in the monolayer due to the change in the weak interlayer interaction. PBE calculations underestimate the band gap significantly, suggesting a value of $0.04 \mathrm{eV}$ for the bulk and $0.9 \mathrm{eV}$ for the monolayer. We thus expect also the calculated band gap in isolated chains to lie below the experimental value and to decrease due to interchain interaction in $2 \mathrm{D}$ chain assemblies. In helical coils, a further reduction, which should furthermore depend on the coil radius, is expected due to improved screening of the electron-hole interaction. In view of this reasoning, it is not surprising that our calculated band gap values lie rather close to the value of $1.95 \mathrm{eV}$ that has been observed in fibrous red phosphorus. 27

Further electronic structure changes induced by coil deformation may open a wide range of applications. Similar to bulk black phosphorus, where changes in the interlayer distance $d$ modify the band gap $E_{g}$ significantly, we find that axial compression of the coil also modifies the band gap. At the optimum value $0.73 \mathrm{~nm}$ for the inter-coil distance $d$, defined in Fig. 2(b), the gap is direct and $E_{g}=1.8 \mathrm{eV}$ according to Fig. 3(b). Reducing $d$ to $0.6 \mathrm{~nm}$ turns the gap indirect and reduces its value to $\approx 0.8 \mathrm{eV}$. Further reduction to $d=0.5 \mathrm{~nm}$ turns the coil metallic. Increasing the inter-chain distance to $d=0.8 \mathrm{~nm}$ opens the gap to $E_{g} 2.0 \mathrm{eV}$, while maintaining its direct character. Of course, these changes in the fundamental band gap may only be exploited inside semiconducting nanotubes, such as $\mathrm{BN}$, which have even 
larger band gaps.

\section{Conclusions}

In conclusion, we have identified computationally an unusually stable helical coil allotrope of phosphorus. Our $a b$ initio Density Functional Theory calculations indicate that the uncoiled, isolated straight $1 \mathrm{D}$ chain is equally stable as a monolayer of black phosphorus dubbed phosphorene. The coiling tendency and the attraction between adjacent coil segments add an extra stabilization energy of $\approx 12 \mathrm{meV} /$ atom to the coil allotrope, similar in value to the $\approx 16 \mathrm{meV} /$ atom inter-layer attraction in bulk black phosphorus. Thus, the helical coil structure is essentially as stable as black phosphorus. In view of the low beam rigidity of the chain and the large inter-chain interaction, a structure consisting of nested coaxial coils should be even more stable than bulk black phosphorus, the most stable phosphorus allotrope known to date. With an optimum radius of $2.4 \mathrm{~nm}$, the helical coil of phosphorus may fit well and even form inside wide carbon nanotubes. We find the coiled $P$ structure to be a semiconductor with a direct gap exceeding $1.8 \mathrm{eV}$. The size and character of the band gap can further be modified by small structural changes in the coil.

\section{Methods}

Global search for small structural fragments of phosphorus was performed using Adaptive Genetic Algorithms based on a tight-binding Hamiltonian with universal parameters. ${ }^{14}$ Suitable structural candidates were then optimized using $a b$ initio density functional theory (DFT) as implemented in the SIESTA ${ }^{28}$ code to obtain insight into the equilibrium structure, stability and electronic properties of phosphorus structures reported in the main manuscript. All isolated structures, including infinite 1D chains and bent chain segments, have been represented using periodic boundary conditions and separated by a $15 \AA$ thick vacuum region in all directions. We have used the Perdew-Burke-Ernzerhof (PBE) ${ }^{29}$ or alternately the Local Density Approximation (LDA) forms of the exchange-correlation functional, norm-conserving Troullier-Martins pseudopotentials, 32 and a local numerical double- $\zeta$ basis including polarization orbitals. The Brillouin zone of periodic structures has been sampled by a fine grid $^{33}$ of $12 \times 1 \times 1 \mathrm{k}$-points for $1 \mathrm{D}$ structures and $12 \times 8 \times 1 \mathrm{k}$-points for $2 \mathrm{D}$ structures. We found our basis, our $k$-point grid, and the mesh cutoff energy of 180 Ry used in the Fourier representation of the self-consistent charge density to be fully converged, providing us with a precision in total energy of $2 \mathrm{meV} / \mathrm{atom}$. All geometries have been optimized using the conjugate gradient method $^{34}$ until none of the residual HellmannFeynman forces exceeded $10^{-2} \mathrm{eV} / \AA$.

Supporting Information Available: A movie depicting schematically the formation mechanism of a helical coil of phosphorus inside a cylindrical cavity.

This material is available free of charge via the Internet at http: / / pubs.acs.org//

\section{Author Information}

\section{Corresponding Author}

*E-mail: tomanek@pa . msu . edu

\section{Notes}

The authors declare no competing financial interest.

Acknowledgement We acknowledge the assistance of Garrett B. King with the graphical representation of evolving structures. This study was supported by the NSF/AFOSR EFRI 2-DARE grant number \#EFMA-1433459. Computational resources have been provided by the Michigan State University High Performance Computing Center.

\section{References}

(1) Elemental phosphorus was first extracted from human urine by German alchemist Hennig Brand around 1669.

(2) Thurn, H.; Krebs, H. Angew. Chem. Internat. Edit. 1966, 5, 1047-1048.

(3) Thurn, H.; Krebs, H. Acta Crystallogr. Sect. B 1969, 25, 125-135. 
(4) Hittorf, W. Annalen der Physik und Chemie 1865, 202, 193-228.

(5) Ruck, M.; Hoppe, D.; Wahl, B.; Simon, P.; Wang, Y.; Seifert, G. Angew. Chem. Int. Ed. 2005, 44, 7616-7619.

(6) Hultgren, R.; Gingrich, N. S.; Warren, B. E. J. Chem. Phys. 1935, 3, 351-355.

(7) Bridgman, P. W. J. Am. Chem. Soc. 1914, 36, 1344-1363.

(8) Zhu, Z.; Tománek, D. Phys. Rev. Lett. 2014, 112, 176802.

(9) Zhang, J. L.; Zhao, S.; Han, C.; Wang, Z.; Zhong, S.; Sun, S.; Guo, R.; Zhou, X.; Gu, C. D.; Yuan, K. D.; Li, Z.; Chen, W. Nano Lett. 2016, 16, 4903-4908.

(10) Haeser, M.; Schneider, U.; Ahlrichs, R.

(11) Jones, R. O.; Seifert, G. J. Chem. Phys 1992, 96, 7564-7572.

(12) Seifert, G.; Jones, R. O. Z. Phys. D 1993, 26, 349-351.

(13) Pfister, D. et al. Adv. Mater. 2016, 9783 Ü9791.

(14) Liu, D.; Jiang, J.; King, G. B.; Moskovtsev, K.; Tomanek, D. in preparation.

(15) Pfitzner, A.; Bräu, M. F.; Zweck, J.; Brunklaus, G.; Eckert, H. Angew. Chem. Int. Ed. 2004, 43, 4228-4231.

(16) Bachhuber, F.; von Appen, J.; Dronskowski, R.; Schmidt, P.; Nilges, T.; Pfitzner, A.; Weihrich, R. Angew. Chem. Int. Ed. 2014, 53, 11629-11633.

(17) Grotz, C.; Schäfer, K.; Baumgartner, M.; Weihrich, R.; Nilges, T. Inorg. Chem. 2015, 54, 10794-10800.

(18) Karttunen, A. J.; Linnolahti, M.; Pakkanen, T. A. Chem. Eur. J. 2007, 13, 52325237.

(19) Maceri, A. Theory of Elasticity; Springer: Berlin, 2010.
(20) Shulenberger, L.; Baczewski, A. D.; Zhu, Z.; Guan, J.; Tomanek, D. Nano Lett. 2015, 15, 8170-8175.

(21) Bandow, S.; Takizawa, M.; Hirahara, K.; Yudasaka, M.; Iijima, S. Chem. Phys. Lett. 2001, 337, 48-54.

(22) Zhang, J.; Feng, Y.; Ishiwata, H.; Miyata, Y.; Kitaura, R.; Dahl, J. E. P.; Carlson, R. M. K.; Shinohara, H.; Tománek, D. ACS Nano 2012, 6, 8674-8683.

(23) Zhang, J.; Zhu, Z.; Feng, Y.; Ishiwata, H.; Miyata, Y.; Kitaura, R.; Dahl, J. E. P.; Carlson, R. M. K.; Fokina, N. A.; Schreiner, P. R.; Tománek, D.; Shinohara, H. Angew. Chem. Int. Ed. 2013, 52, 3717-3721.

(24) Liu, H.; Neal, A. T.; Zhu, Z.; Luo, Z.; Xu, X.; Tomanek, D.; Ye, P. D. ACS Nano 2014, 8, 4033-4041.

(25) Jinying Zhang (private communication).

(26) Liu, D.; Every, A. G.; Tománek, D. Phys. Rev. B 2016, 94, 165432.

(27) Fasol, G. J. Phys. C: Solid State Phys. 1985, 18, 1729-1741.

(28) Artacho, E.; Anglada, E.; Dieguez, O.; Gale, J. D.; Garcia, A.; Junquera, J.; Martin, R. M.; Ordejon, P.; Pruneda, J. M.; Sanchez-Portal, D.; Soler, J. M. J. Phys. Cond. Mat. 2008, 20, 064208.

(29) Perdew, J. P.; Burke, K.; Ernzerhof, M. Phys. Rev. Lett. 1996, 77, 3865-3868.

(30) Ceperley, D. M.; Alder, B. J. Phys. Rev. Lett. 1980, 45, 566-569.

(31) Perdew, J. P.; Zunger, A. Phys. Rev. B 1981, 23, 5048-5079.

(32) Troullier, N.; Martins, J. L. Phys. Rev. B 1991, 43, 1993-2006.

(33) Monkhorst, H. J.; Pack, J. D. Phys. Rev. B 1976, 13, 5188-5192.

(34) Hestenes, M. R.; Stiefel, E. J. Res. Natl. Bur. Stand. 1952, 49, 409-436. 